\title{
Pulmonary complications of oxygen therapy
}

\author{
R. ROWLAND AND C. G. H. NEWMAN \\ From St Thomas's Hospital, London
}

SYNOPSIS Three cases of the neonatal respiratory distress syndrome are described. The babie were treated by prolonged oxygen given by intermittent positive pressure respiration after endog tracheal intubation. Two of the infants died and at necropsy the lungs in both cases were conit solidated. Both showed widespread haemorrhagic exudate and interstitial oedema. Early organiz $\alpha_{0}^{N}$ tion of the exudate was apparent in one case. The third infant survived but developed a similat exudative condition and a 'honeycomb' lung. It is suggested that many of the changes found in the lungs of these cases could be attributed to the toxicity of oxygen.

The prolonged use of oxygen in tensions above that found in air has been employed in space research and deep sea diving. In medicine it is used in radiotherapy and in intensive care of both adults and infants.

The toxic effects of oxygen depend on its partial pressure and on individual and species sensitivity (Bean, 1965). At very high tensions, above 3 atmospheres, the primary disturbances are noted in the nervous system while at lower pressures, up to 2 atmospheres, the lung appears to react first (Ledingham, 1967). The effect of oxygen at 3.7 atmospheres' tension on the central nervous system in man is well illustrated by the work of Donald (1947) who showed that the tolerance of 36 subjects varied from seven to 96 minutes. Convulsions and twitching were the first symptoms to develop. At lower tensions minor constriction of the vessels of the adult cerebrum and retina may occur as well as retrolental fibroplasia in premature infants (Klaus and Meyer, 1966).

That the lung could be affected by lower tensions of oxygen was illustrated by Lorraine Smith in 1899 who showed that mice exposed to $79 \%$ of an atmosphere oxygen tension developed pulmonary congestion and consolidation and about half the animals died.

In human volunteers at oxygen tensions of 1 atmosphere the average tolerance was from 53 to 75 hours, while the onset of symptoms varied from six to 30 hours. The first symptom in most cases was substernal distress (Welch, Morgan, and Clamann, 1963).

Nash, Blennerhassett, and Pontoppidan (1967) in a controlled trial showed that in adults after proReceived for publication 3 May 1968. longed artificial ventilation morphological changes developed in the lungs. The changes were unrelate to the use of the ventilator as such but were core related with the ventilator delivering a high cono centration of oxygen. The pulmonary changes were characterized by an early exudative phase in whic there was haemorrhagic exudation into the lung and a late proliferative phase characterized by oedemap alveolar cell metaplasia, and interstitial fibrosis.

Shanklin and Wolfson (1967) found pulmonar haemorrhage in a significantly greater number of neonates who had received early or continuous oxygen therapy as compared with control groups. More recently the relation of oxygen therapy to lung haemorrhage has been discussed (British Medicat Journal, 1968). It was concluded that the risk was. such that regular monitoring of alveolar and blood gas tension should be done so that dangerously high concentrations could be avoided if possible.

Studying neonates with the respiratory distress syndrome treated with oxygen and intermitter positive pressure respiration, Northway, Rosan, and Porter (1967) described a pattern of lung pathology and called it 'bronchopulmonary dysplasia'. It was confined, in their series, to infants surviving aftect more than 150 hours' exposure to high tensions of oxygen (up to 1 atmosphere) while on a ventilatore Their cases showed exudate in the alveoli, metio plasia of the bronchiolar epithelial cells, interstitiaf oedema, and fibrosis. Hawker, Reynolds, and Taghizadeh (1967) reported five infants who die with similar lung changes after five to 13 days o\& positive pressure respiration with high-tensio oxygen for severe hyaline membrane disease Fibrosis was a prominent feature. 
We describe below three infants who suffered an exudative lung condition, fatal in two of them, which complicated an idiopathic respiratory distress syndrome after as little as $\mathbf{4 2}$ hours on high-oxygen and respirator treatment. We consider that this pulmonary exudative lesion bore a sufficient resemblance to the descriptions of oxygen damage to the lung as to suggest a causal relationship.

\section{MATERIAL AND METHODS}

The three infants were born at between 31 and $32 \frac{1}{2}$ weeks gestation. All three developed severe respiratory distress which was treated with intravenous fluid, giucose, alkali, and antibiotics. Intermittent positive pressure respiration was started when it became clear that the infants could not survive without further assistance. Chilling was avoided. The incubator temperature was set to produce a skin temperature (electronic temperature probe) of 96 to $97^{\circ} \mathrm{F}$. Oxygen concentrations were monitored with a Beckman oximeter. The respirator used was a Bird VIII model fitted with an infants' $Q$ circle and increased capacity humidifier. The lowest effective inspiratory pressure was used. At the flow rates used for neonates and with oxygen to drive the ventilator only $100 \%$ or $92 \%$ oxygen could be given. Lower concentrations could not be given except by running the respirator on air alone. Cylinders with other oxygen concentrations were not used in the unit at that time.

CASE 1 The mother was aged 35 and had one living infant and six previous miscarriages.

Labour began spontaneously at 32 weeks' gestation, and a male infant weighing $5 \mathrm{lb}$ was delivered. $\mathrm{He}$ breathed within 30 seconds of delivery but developed the respiratory distress syndrome over the first five hours of life. The $\mathrm{pCO}_{2}$ rose to $105 \mathrm{~mm} \mathrm{Hg}$ and there was respiratory arrest in $80 \%$ oxygen. The infant was intubated and put on intermittent positive pressure respiration from the fifth to the 51st hour. Secretions were troublesome towards the end of this period. He was extubated for a trial period of four hours but his condition suddenly deteriorated and necessitated reintubation because of laryngeal spasm. A further trial of extubation at 73 hours of age again failed. Tracheostomy was performed and the child was maintained on intermittent positive pressure respiration until asphyxia from progressive pulmonary insufficiency supervened at 5 days 4 hours. The physical signs resembled those of pulmonary oedema.

From birth the child was on colistin and penicillin.

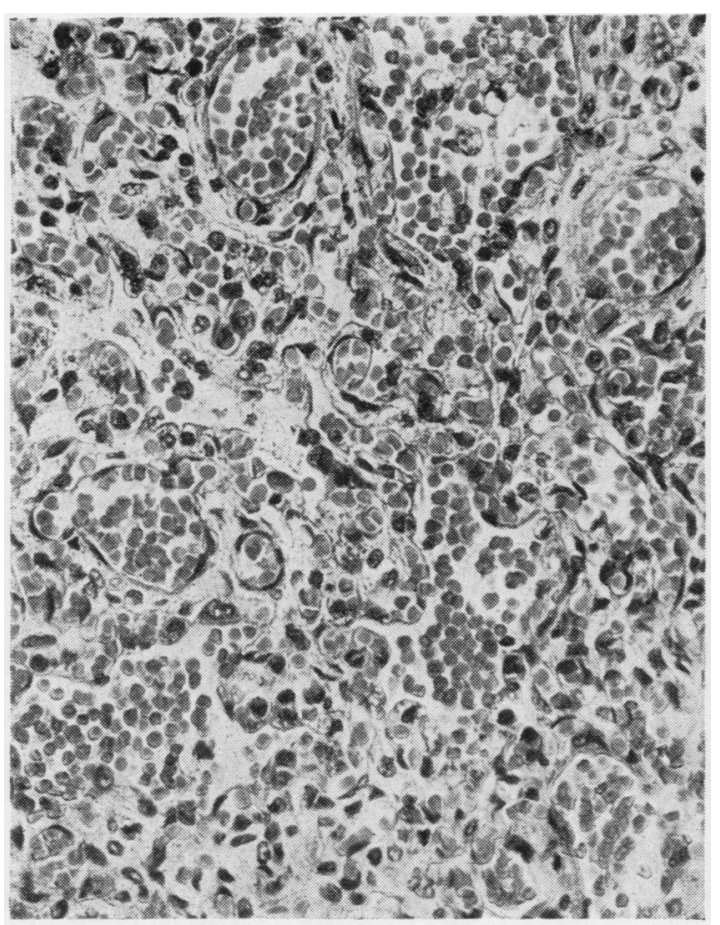

FIG. 1 .

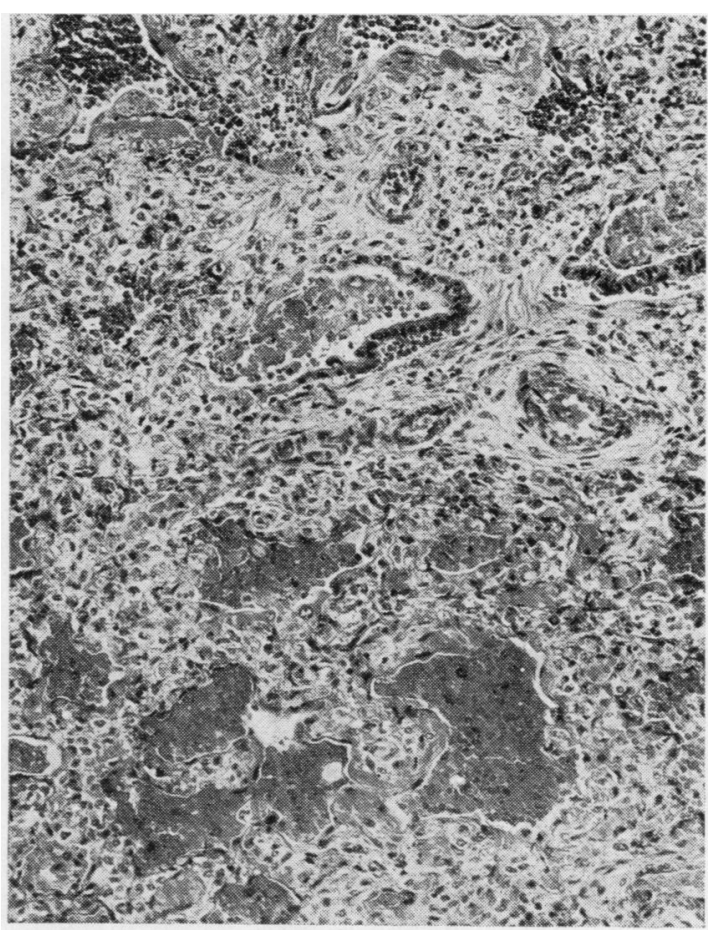

FIG. 2 .

FIG. 1. The alveolar capillaries are dilated and there is haemorrhage in the alveoli.

FIG. 2. The alveoli are filled with haemorrhage in some areas and granular exudate in others. Hyaline membranes are visible. 


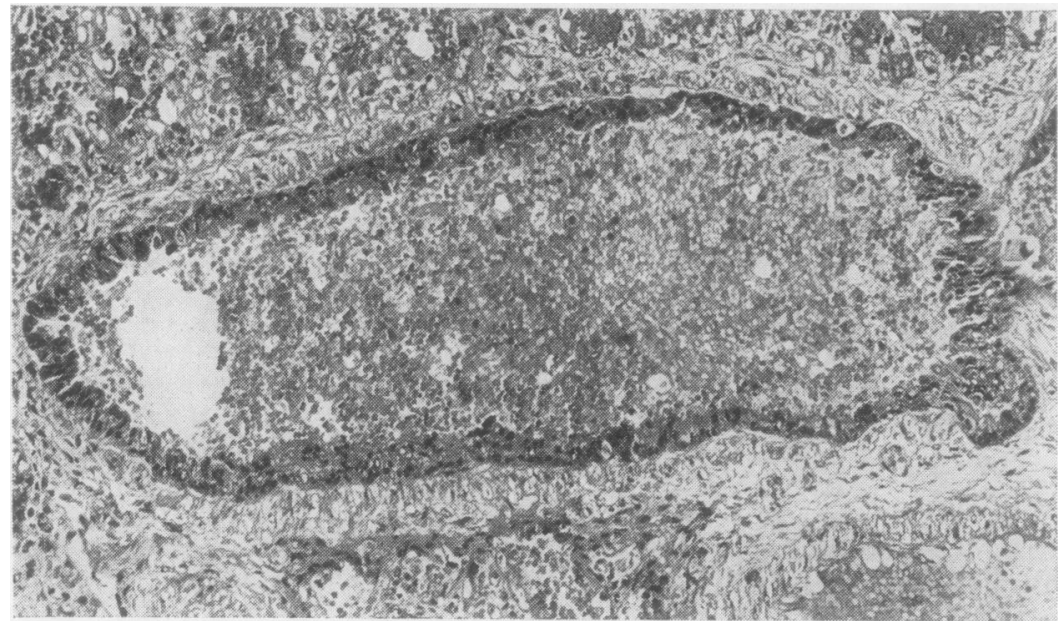

FIG. 3. The bronchus is filled with haemorrhagic exudate. The lining epithelium is atypical.

FIG. 4. There is widespread filling of the alveoli by exudate, and $\vec{\omega}$ overdistension with fusion of adjacent alveoli in some areas.

FIG. 3 .

Necropsy The trachea showed some crusting of the mucosa below the tracheostomy.

The lungs were consolidated and deep purple. The pleural cavities contained a little clear fluid.

There were no other significant macroscopic postmortem findings.

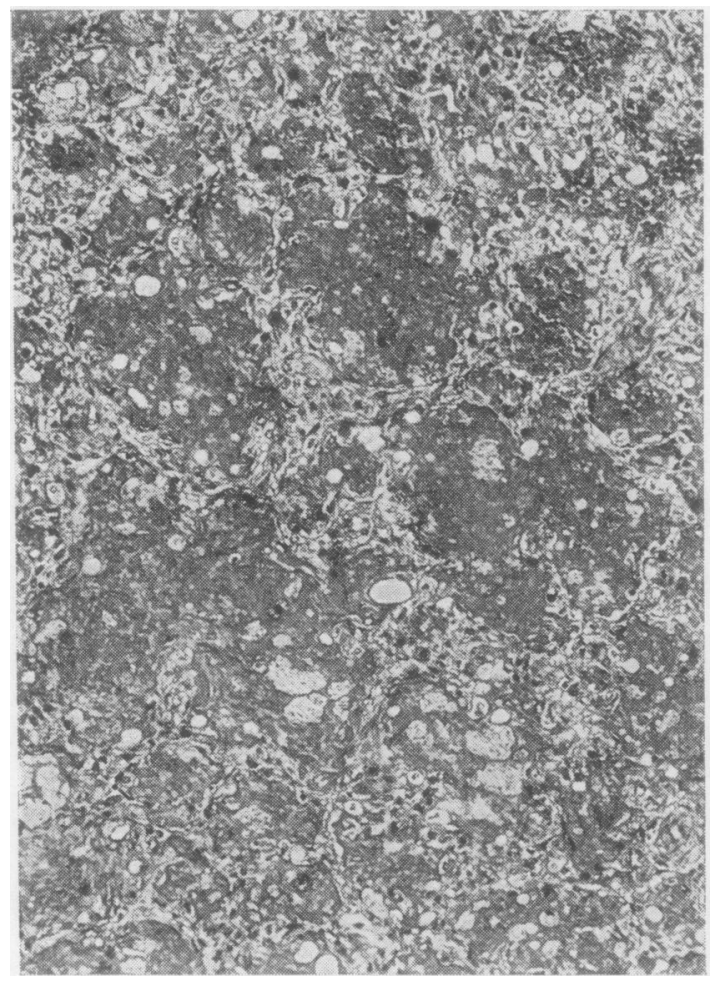

FIG. 4.
The bronchi were filled with homogeneous eosinophil exudate. The alveoli showed widespread congestion and dilatation of capillaries (Fig. 1). In some areas the alveo( were filled by haemorrhage and in others by granuláp eosinophilic exudate (Fig. 2). There were residual hyaline membranes apparent but almost no inflammatory celf Interstitial oedema was apparent in some areas.

CASE 2 The mother, a primipara aged 35, developed severe preeclamptic toxaemia which necessitated caesarian section at 31 weeks' gestation. The infant was male and weighed $2 \mathrm{lb} 11 \mathrm{oz}$ at birth.

From birth he was unable to establish adequat respiration and was therefore intubated and maintained on intermittent positive pressure respiration with $100 \%$ oxygen to 42 hours. Between 42 and 58 hours it w⿳亠口冋亍 possible to maintain oxygenation, running the respiratof on air. Then the child was extubated and maintained his own respiration until death at 9 days. The oxygen coo centration in the incubator was raised from $50 \%$ a extubation to $95 \%$ during the last six days when pro gressive dyspnoea was accompanied by widespread crepitations and increasing pulmonary insufficiency un death.

The child was on a vigorous wide-spectrum antibiot regime, which included ampicillin, orbenin, and colistin by injection and colistin by aerosol. Repeated sputug cultures were sterile.

Necropsy The larynx, trachea, and bronchi were filled with bloodstained mucoid material. The pleura cavities contained a few millilitres of bloodstained flui The lungs were dark purple and completely consolidateon They sank in water.

There were petechial haemorrhages on the viscer pericardium but no other significant postmortem finding

The bronchi were filled with eosinophilic exudate containing numerous red blood cells, and the lining cels seemed atypical (Fig. 3). Residual hyaline membranes were visible. The capillaries of the alveolar walls and septa were dilated. The smaller bronchi, dilated alveolag 


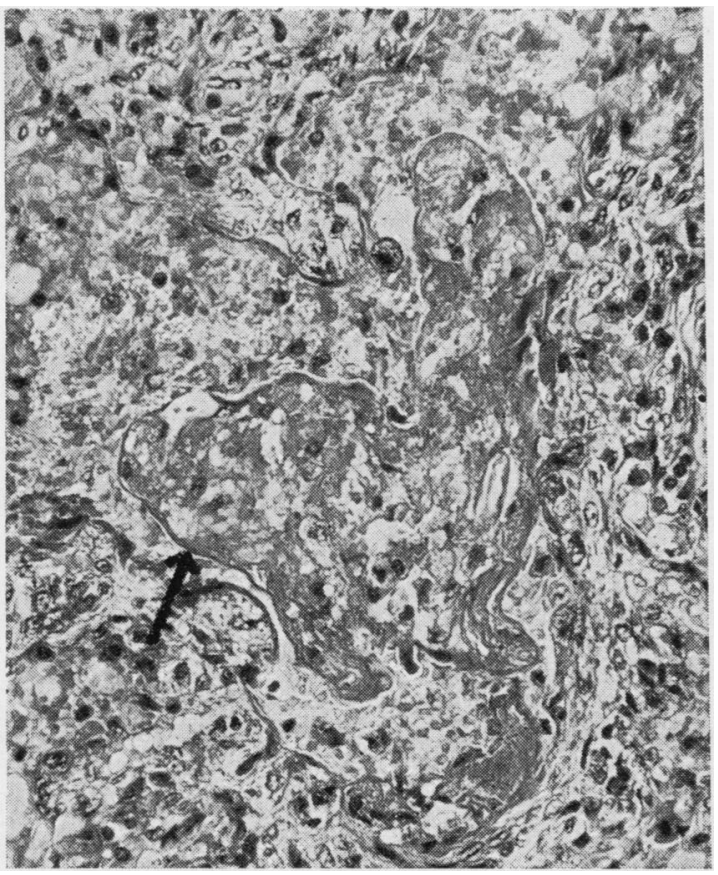

FIG. 5. A mass of exudate is covered by a flattened layer of cells (arrow) continuous with the lining epithelium of the alveolus - an early stage of organization.

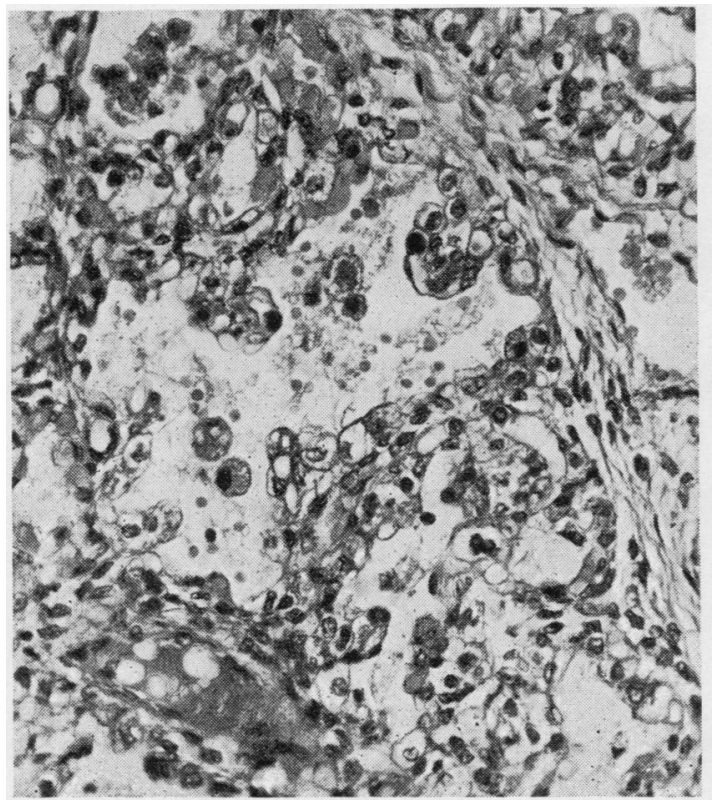

FIG. 6. There is cubical metaplasia of the alveolar lining cells and foamy macrophages are present in the alveoli. There is oedema of one septum.

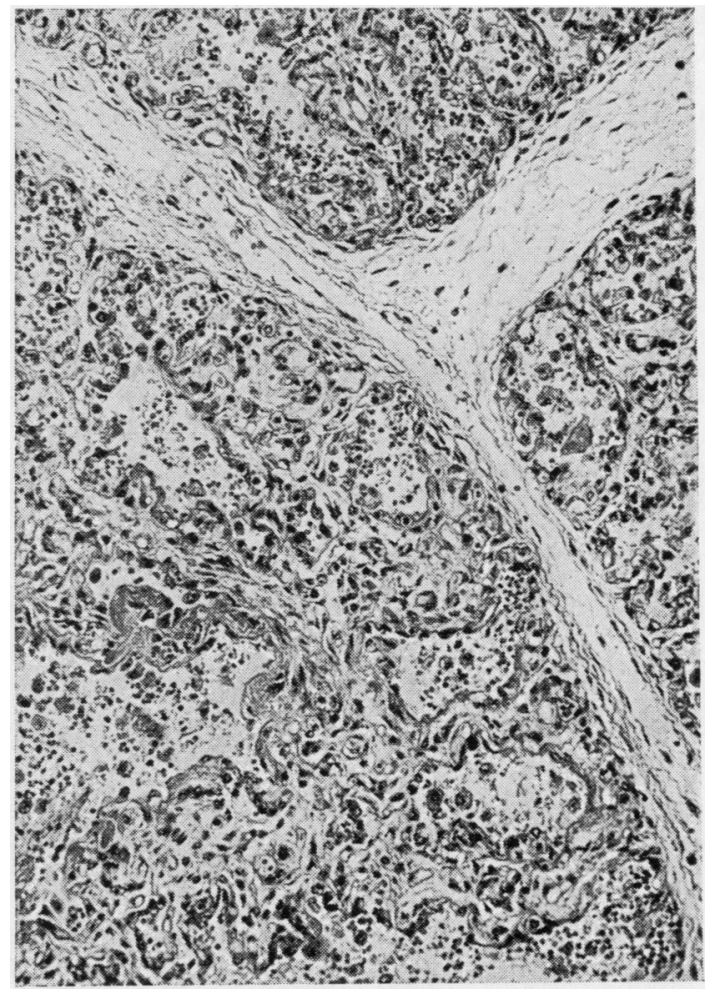

FIG. 7. The interalveolar walls are widened. There is oedema of the septa and hyaline membranes are present.

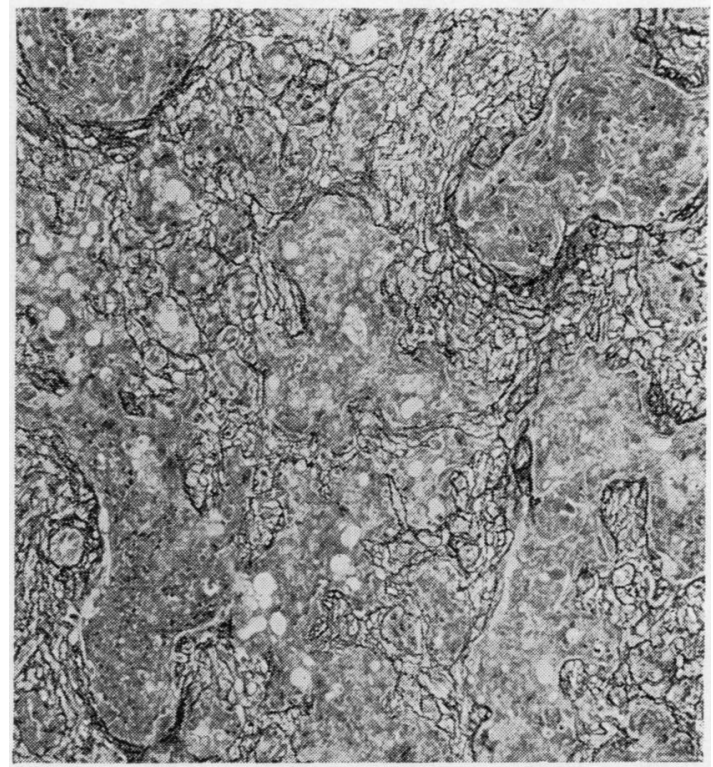

FIG. 8. A reticulin stain showing the increase in reticulin. 
ducts, and alveoli contaitied exudate which varied from place to place. In some areas there was eosinophilic exudate overlying hyaline membranes and in other areas the exudate contained a large amount of blood. Some alveoli were overdistended by exudate, the interalveolar walls breaking down to form abnormally distended, exudate-filled spaces (Fig. 4).

In some areas masses of exudate were covered by a flattened layer of cells continuous with the alveolar lining cells. In this way the exudate appeared to become incorporated into the wall (Fig. 5). Where there was less exudate there was cubical metaplasia of the alveolar lining cells and foamy macrophages were apparent in the alveolar spaces (Fig. 6). Apart from these alveolar macrophages there were no inflammatory cells apparent in the lung sections examined. The interalveolar septa were wide and there was interstitial oedema (Fig. 7). Reticulin stains showed an apparent increase in fibre in the widened septa (Fig. 8), possibly due to atelectasis.

CASE 3 The infant was born at $32 \frac{1}{2}$ weeks' gestation. The birth weight was $3 \mathrm{lb} 13 \mathrm{oz}$ and she breathed within 30 seconds of delivery.

She became shocked at 5 hours and respiration was shallow and rapid. Soon afterwards she required intubation and was put on intermittent positive pressure respiration with $100 \%$ oxygen. At 48 hours there was excessive mucoid secretion but adequate ventilation was maintained nevertheless.

The infant continued on the respirator until she was 12 days old when she was extubated and managed at first to maintain adequate respiration unaided but over the next five days she required short periods of assisted respiration. Excessive mucoid secretion continued to be troublesome.

A chest radiograph showed the reticulo-granular

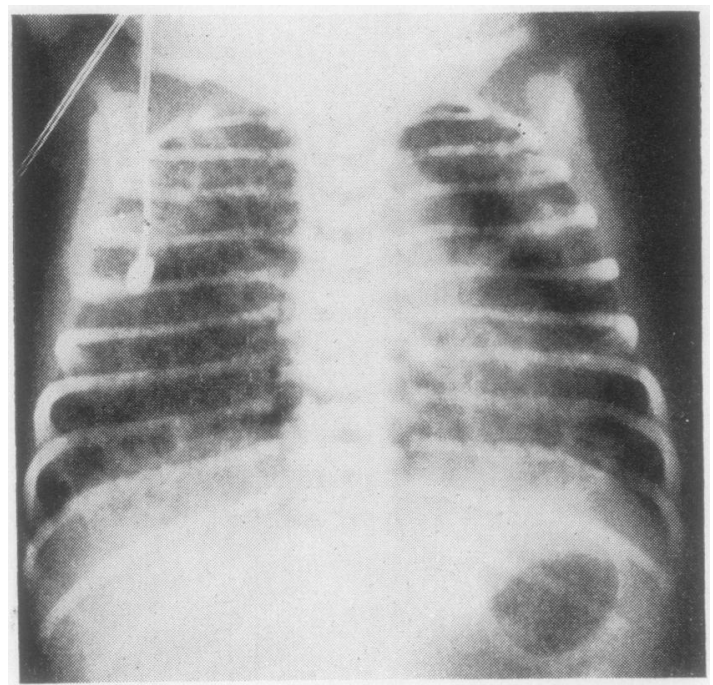

FIG. 9. The chest radiograph at nine days shows a 'honeycomb' appearance. appearance associated with hyaline membrane disease by으 the third day. This was followed by the appearance of a honeycomb lung by the ninth day (Fig. 9). At 6 weeks this honeycomb pattern was still present.

Her ECG showed peaked $P$ waves suggestive of right atrial stress.

The child gradually improved but had repeated ches $\frac{\bar{T}}{\square}$ infections over the next four months. She was unfortu- $\varrho$ nately lost to follow up.

\section{DISCUSSION}

In the first case, the lung showed widespreado haemorrhagic exudation. The dilated, congested capillaries suggested that the haemorrhage and exudation had come from the lung and was not aspirated. At this stage there was little apparent reaction in the lung parenchyma. The hyaline membranes were compatible with resolving hyaline membrane disease.

The second case showed similar congestion and exudation. There was, however, evidence of early organization of the exudate characterized by the growth of flattened cells over masses of the exudates and so incorporating them into the alveolar wall. Ifo this baby had survived some residual fibrosis could have been expected from organization of this amount of exudate. Both these cases were exposed to high tension oxygen for a much shorter time thang the 150 hours noted by Northway et al (1967).

In the final case the chest radiographs show the development of a honeycomb lung from the radio윽 logical appearances of hyaline membrane disease: $-\bar{r}$ The most marked changes occurred during the periof of the most intensive care.

These cases illustrate an illness where hyaline membrane disease is followed by an exudative reaction which may become haemorrhagic, and then may be absorbed or organized. Its organization maye prevent complete resolution, and a diffuse inter stitial pulmonary fibrosis could well follow. This pattern does not generally occur in hyaline mem $\rightarrow$ brane disease, where complete resolution is likely it the baby survives at all.

It is not possible to be dogmatic about the cause of the prolonged pulmonary abnormality seen in infants with severe hyaline membrane disease whose lives have been extended by treatment with res? pirators.

The first infant died at just over 5 days of age from what appeared clinically to be progressive pulmonary oedema. The hyaline membrane disease had abatedo but continued respirator treatment was necessary because of airway obstruction and secretions. Case 2 initially improved as well, according to the expected pattern of simple hyaline membrane disease, but then began to deteriorate from the sixth day, with 
increasingly wet lungs and progressive pulmonary insufficiency. This initial improvement makes it less likely that a very severe and prolonged form of hyaline membrane disease was responsible for the whole picture. In the third infant, severe pulmonary abnormality persisted, but excessive secretions atypical of hyaline membrane disease, and not apparently due to infection, were at least partly responsible. All the infants received wide-spectrum antiobiotics and in the two examined at necropsy there was no histological evidence of infection.

The Wilson-Mikity syndrome describes the delayed clinical and radiological changes occurring in the lungs of very small premature infants. Pulmonary exudation is not a prominent feature (Burnard, 1966), but exudate, even blood, has been found microscopically in the alveoli of some necropsied cases. This could have been an effect of the oxygen treatment that these infants received. The surviving infant in this report developed radiological changes very like those found in the Wilson-Mikity syndrome, but the changes following oxygen damage can be similar. The surviving infant was

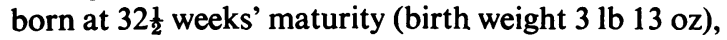
which is more mature and heavier than many of the infants described in reports of the Wilson-Mikity syndrome, but this condition may nevertheless have played a part.

We consider that oxygen damage is the likeliest explanation for the changes that occurred. The experimental evidence (Kistler, Caldwell, and Weibel, 1967; Smith, 1899; Schaffner, Felig, and Trachtenberg, 1967), clinical reports such as those of Northway et al (1967), Nash et al (1967), Hawker et al (1967), Shanklin and Wolfson (1967), and our own experience add up to strong circumstantial evidence incriminating high-tension oxygen therapy.

Troublesome secretions do not occur equally in every case or in every centre, and this may relate to the different respirators used and the different tensions of oxygen administered.

Accepting that the changes in our cases resembled the pathology produced experimentally in animals, case 1 was in the early exudative phase; case 2 showed exudation but early organization also; and case 3 showed the radiological pattern of developing interstitial fibrosis, of which the cases described by Hawker et al (1967) and Northway et al (1967) showed the pathology. It may be relevant that all the three infants we describe were treated with hightension oxygen within six hours of birth, a period of possibly enhanced vulnerability, as suggested by Shanklin and Wolfson (1967).

The mechanism of oxygen damage to the lung has not been fully elucidated. Kistler et al (1967) studied the early effects of 1 atmosphere of oxygen on rats and did electron microscopic studies on groups of animals exposed for varying periods up to three days. They showed that the main changes occurred between the second and the third day and were characterized by the accumulation of oedema fluid in the interstitium accompanied by massive capillary endothelial destruction, fibrin and cells subsequently exuding into the interstitium and alveoli. The basic lesion appeared to be damage to the capillary alveolar endothelium.

Schaffner et al (1967), also using rats, showed that after exposure to $700 \mathrm{~mm} \mathrm{Hg}$ oxygen for up to 10 days, capillary damage, interstitial oedema, and fibrosis were apparent in the lungs.

In an attempt to integrate the various pathological effects on the lung which have been attributed to oxygen toxicity we have drawn up the scheme shown in Figure 10. These effects, superimposed on the

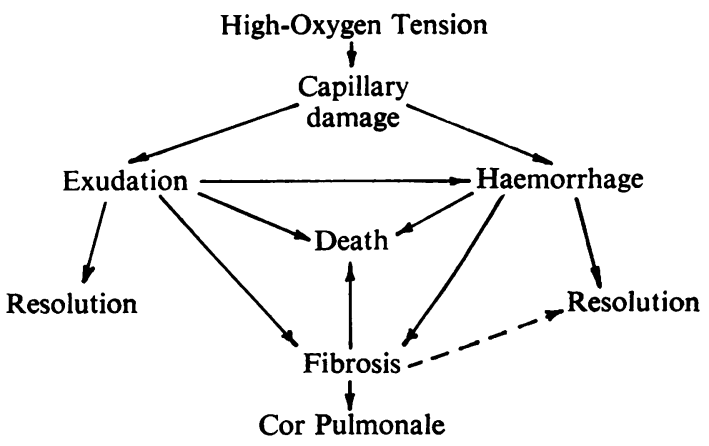

FIG. 10. The effects of high-oxygen tension on the lung.

conditions for which high-tension oxygen is used in treatment, can explain a number of otherwise atypical features. The conditions known to be most commonly associated with massive neonatal pulmonary haemorrhage, to which oxygen therapy has recently been added (Shanklin and Wolfson, 1967), include major cerebral abnormality including kernicterus, low birth weight for dates (2 SD below the weight expected for the period of gestation), and the cold syndrome. Brain abnormality is present in the surviving infant, but could well be the result of repeated severe episodes of asphyxia from airway obstruction due to secretions. The other conditions did not occur. There was no evidence of sepsis during life or at necropsy, so it seems unlikely that infection played a significant part in producing the exudation.

In conclusion, we feel that continuous oxygen at 1 atmosphere is toxic to the lung in some newborn infants. We agree with the views expressed in a leading article in the British Medical Journal (1968) that 
careful monitoring of blood gas tensions is required when high-tension oxygen is used. To use a dose greater than the minimum necessary to provide adequate arterial oxygenation may be dangerous but can easily happen with less controlled oxygen therapy. Respirators unable to deliver sufficiently variable concentrations of oxygen must be modified, run from variable gas sources, or changed for the more versatile machines. The actual, rather than the nominal, concentrations of gas delivered must be known, and the lowest effective concentrations of oxygen used. There may be a limiting factor because some infants require potentially toxic oxygen exposure for survival, and in these cases pharmacological measures to reduce the toxic effect of oxygen on the lung (Bean, 1965) may yet pay dividends.

We wish to thank Dr J. R. Tighe for his unfailing help and constructive criticism. We would also like to thank Professor H. Spencer and Dr R. John for their assistance, and Dr D. C. Cottom for permission to study the newborn infants under his care.

\section{REFERENCES}

Bean, J. W. (1965). Ann. N.Y. Acad. Sci., 117, 745.

British Medical Journal (1968). Leader, 1, 397.

Burnard, E. D. (1966). Pediat. Clin. N. Amer., 13, 999.

Donald, K. W. (1S47). Brit. med. J., 1, 667.

Hawker, J. M., Reynolds, E. O. R., and Taghizadeh, A. (1967). ڤ Lancet, $1,75$.

Kistler, G. S., Caldwell, P. R. B., and Weibel, E. R. (1967). J. Cell $\vec{\circ}$ Biol., 32, 605.

Klaus, M., and Meyer, B. P. (1966). Pediat. Clin. N. Amer., 13, 731. $\vec{\omega}$

Ledingham, I. M. (1967). Sci. Basis Med. Ann. Rev., 90.

Nash, G., Blennerhassett, J. B., and Pontoppidan, H. (1967). New Engl. J. Med., 276, 368. Northway, W. H., Jr, Rosan, R. C., and Porter, D. Y. (1967). Ibid., N
276, 357.

Schaffner, F., Felig, P., and Trachtenberg, E. (1967). Arch. Path., N $83,99$.

Shanklin, D. R., and Wolfson, S. L. (1967). New Engl. J. Med., 277, 833.

Smith, J. L. (1899). J. Physiol. (Lond.), 24, 19.

Welch, B. E., Morgan, T. E., and Clamann, H. G. (1963). Fed. Proc., 22, 1053. 